Poster Section

\title{
Homeopathic dilutions of TNF-alfa in psoriasis and rheumatoid arthritis
}

\author{
Adrian Alecu ${ }^{1}$, Dumitru Gafitanu², Mariana Alecu³ \\ Diana Popa ${ }^{3}$, Ioana Cridon ${ }^{3}$ \\ 1 University of Medicine and Pharmacy, Targu-Mures, Romania \\ 2 University of Medicine and Pharmacy "Gr. T. Popa", Iasi, Romania \\ ${ }^{3}$ Lotus Life Integrative Medicine Center, Targu-Mures, Romania
}

\begin{abstract}
Aim: Clinical and biological studies in the past years underlined the proinflammatory action of the citokine Tumoral Necrosis Factor in the pathophysiolology of psoriasis, psoriatic arthritis, rheumatoid arthritis. Knowing that the high dilution of substances can have an inverted effect, our hypotesis was that dillution made of TNF-alfa can decrease the clinical manifestation of such diseases.
\end{abstract}

Materials and method: We included as a pilot group 10 patients presented in Lotus Life Integrative Medicine Center or Profamilia Medical Center Iasi with psoriasis and rheumatoid arthritis previously diagnosed by specialists, under speciality treatment with insufficient results and patients who deliberately expressed their preference for alternative treatment. We decided to exclude the patients which during the study could present aggravation of symptoms.

The treatment protocol consisted in the administration under the tongue of the dilution of TNF-alfa $9 \mathrm{CH}$ in liquid form, 12 drops twice a day for at least 3 months. This was prepared diluting from Guna TNF-alfa $4 \mathrm{CH}$ in distiled water, completing with $30 \%$ of alcohol in the last solution. Patients under other medications continued to take their previous treatment unchanged. We called the patients for follow-up after 3 months. We took the written consent from the patients and the approval of the Ethical Committee of the University of Medicine and Pharmacy "Gr. T. Popa" of Iasi.

Results: To analize the results we followed the subjective evolution of the patients, the total surface and thickness of eruptions in psoriasis patients and the pain intensity and stiffness in arthritis patients. Under this conditions, all the patients showed amelioration, with 2 out of 6 psoriasis patients showing complete clearance of eruptions. No patient reported adverse reactions during the treatment

Conclusions: Even though the group of patients was small and not appropriate for statistical data the presence of a clinical response in all the patients and the absence of adverse reactions sustains the opportunity to extend the research on this subject.

Keywords: TNF-alfa, psoriasis, rheumatoid arthritis 


\section{(c)) EY-NC-ND Licensed to GIRI}

Support: authors declare that this study received no funding

Conflict of interest: authors declare there is no conflict of interest

Received: 01 June 2012; Revised: 10 August 2012; Published: 30 September 2012.

Correspondence author: Adrian Alecu, doctoralecu@yahoo.com

How to cite this article: Alecu A, Gafitanu D, Alecu M, Popa D, Cridon I. Homeopathic dilutions of TNF-alfa in psoriasis and rheumatoid arthritis. Int J High Dilution Res [online]. 2012 [cited YYYY Month dd]; 11(40):204-205. Proceedings of the XXVI GIRI Symposium; 2012 Sep 20-22; Florence (Italy). GIRI; 2012; Available from: http://www.feg.unesp.br/ ojs/index.php/ijhdr/article/view609/611 